\section{Sudden Onset Altered Sensorium: Artery of Percheron Infarct}

\section{Abstract}

Acute onset Altered mental status is a common but clinically challenging emergency due to numerous possibilities with little clinical window and time to treat. The numerous causes include stroke, encephalitis, organ dysfunction, metabolic, endocrine and intoxication. Amongst the vascular causes, one rare but peculiar cause being artery of percheron infarct. Blood supply to the thalamus and brainstem have various anatomic variations. Artery of Percheron is one such variation in which a single arterial trunk arises from proximal segment of one of the posterior cerebral artery (PCA) and bifurcates to supply bilateral paramedian thalami. Occlusion of this artery, although uncommon results in characteristics pattern of symmetric infarction in bilateral medial thalami with or without mesencephalic infarction. We here describe a case of a gentleman who presented with sudden onset altered mental status and skew deviation. After improvement in sensorium characteristic vertical gaze palsy was noted with subtle confusion which recovered. MRI brain revealed characteristic Para median bithalamic infarcts with normal intracranial angiogram. Patient showed a slow and incomplete clinical recovery to conservative management.

Keywords: Altered mental status; Stroke; Artery of percheron

Received: July 17, 2017; Accepted: July 28, 2017; Published: July 31, 2017

\section{Tushar Premraj Raut*, Gaurav Baheti, Anupa Hinduja, Prashant Makhija, Khursheed Ansari and Vivek Sharma}

\author{
Department of Neurology, Seven Hills \\ Hospital, Mumbai, India
}

*Corresponding author:

Tushar Premraj Raut

tushar.27r@gmail.com

Department of Neurology, Seven Hills Hospital, Mumbai, India.

Tel: +919930087434

Fax: +919930087434

Citation: Raut TP, Baheti G, Hinduja A, Makhija P, Ansari K, et al. (2017) Sudden Onset Altered Sensorium: Artery of Percheron Infarct. J Neurol Neurosci. Vol. 8 No. 4:212

\section{Introduction}

Acute onset Altered mental status (AMS) is a common medical emergency occurring due to a myriad of causes with varied clinical presentation manifesting as cognitive disorders, attention disorders, arousal disorders, and decreased level of consciousness [1]. The level of consciousness may vary from mild drowsiness to stupor and even coma. Hence clinical assessment may not be very conclusive and there comes role of neuroimaging and metabolic work up. There are various structural causes like stroke, tumour with raised intracranial pressure, infection (meningitis, encephalitis) and non-structural causes like toxin induced, metabolic, organ dysfunction. Stroke or vascular etiology is commonly encountered cause of acute onset altered mental status. Amongst the vascular cause, one important though rare because being artery of percheron infarct which has peculiar clinical and radiological features which includes bithalamic infarcts with or without midbrain infarcts. Thalamus is primarily supplied by the perforating arteries from the Posterior Communicating Artery and Posterior cerebral artery. The branches on either side include Para median, inferolateral, tuberothalamic and posterior choroidal. Involvement of anomalous Para median artery which arises from P1 segment of PCA often results in bithalamic infarcts. This anatomic variant in which both Para median arteries arise from a single P1 trunk was described by Gerard Percheron and is termed as the Artery of Percheron (AOP) [2]. Studies found that AOP infarction involves around $0.1 \%$ to $0.3 \%$ of all ischaemic strokes of which $22 \%$ to $35 \%$ are associated with thalamic infarction [3]. Clinical features include varying level of consciousness from drowsiness to coma, vertical gaze palsy, memory disturbances. Here the authors describe a case of a gentleman presenting with sudden onset altered consciousness with additional clinical features like gaze palsy with characteristic MRI features.

\section{Case Report}

A 62-year-old male known case of systemic hypertension on regular antihypertensive medications, also a chronic smoker and alcoholic presented with sudden onset altered sensorium. Patient was having his dinner when he collapsed suddenly and was in 
an unresponsive state. There was no preceding headache, fever or any other illness. No obvious seizures noted by bystanders. On the way to emergency he had 2 episodes of vomiting with hematemesis. Patient was also a chronic smoker and alcoholic with recent binge drinking. There was no prior history of stroke, transient ischemic attack or any other neurological or medical illness. When evaluated in emergency department, his vitals were stable. Blood pressure was $140 / 80 \mathrm{mmHg}$, pulse was $80 /$ min regular. Saturation was maintained on room air, sugars were normal. On detailed neurological evaluation, he was found to be stupurous. He winced to pain. Pupils were normal size reacting to light however skew deviation of eyes was noted. Motor response was withdrawal in nature. Glasgow coma scale GCS was 7. Plantar response was extensor. Patient presented at 6 hours from onset of symptoms. His metabolic work up was sent including blood gas analysis which was normal. Patient was immediately subjected to MRI brain with angiogram.

MRI Brain revealed diffusion restricting foci in bilateral thalami and around the third ventricle. There was no midbrain infarct. MR Angiogram didn't reveal any significant stenosis. Thus imaging features were suggestive of artery of percheron infarct (Figure 1). Other test result positive findings included deranged lipid profile with LDL of $178 \mathrm{mg} / \mathrm{dL}$. ECG and $2 \mathrm{~d}$ echo including holter analysis was normal. Patient was hydrated and injection thiamine was started. Also pantoprazole infusion and sucralfate initiated in view of upper gastrointestinal bleed. Single antiplatelet and statins initiated after stabilisation of GI bleed. Patient was managed conservatively in view of active GI bleed. After 24 hours sensorium improved considerably, no further hematemesis. Patient could obey simple commands, mild confusion and amnesia for the events was present. There was vertical gaze palsy with mild gait ataxia. Follow up imaging on discharge revealed established infarcts in thalami and Parathird ventricular region with some haemorrhagic transformation (Figure 2). Patient was discharged on aspirin $150 \mathrm{mg}$, atorvastatin $40 \mathrm{mg}$, oral thiamine and multivitamins and proton pump inhibitor. Patient further improved clinically with residual vertical gaze palsy on last follow up.

\section{Discussion}

Altered mental status which includes level of consciousness from complete loss of consciousness to excitation [4]. Causes include both neurological and non-neurological. Neurological causes include cerebrovascular disease, infection, seizure, space occupying lesion. Non neurological causes include drugs and substance intoxication, infection, metabolic endocrine abnormalities, trauma, cancer, and system/organ dysfunction. Neurological causes being more common and amongst it stroke is an important and treatable cause if timely intervened. Amongst various strokes, a peculiar clinical presentation occurs due to artery of percheron infarct. There are variations in the blood supply to the thalamus most commonly the thalamoperforators

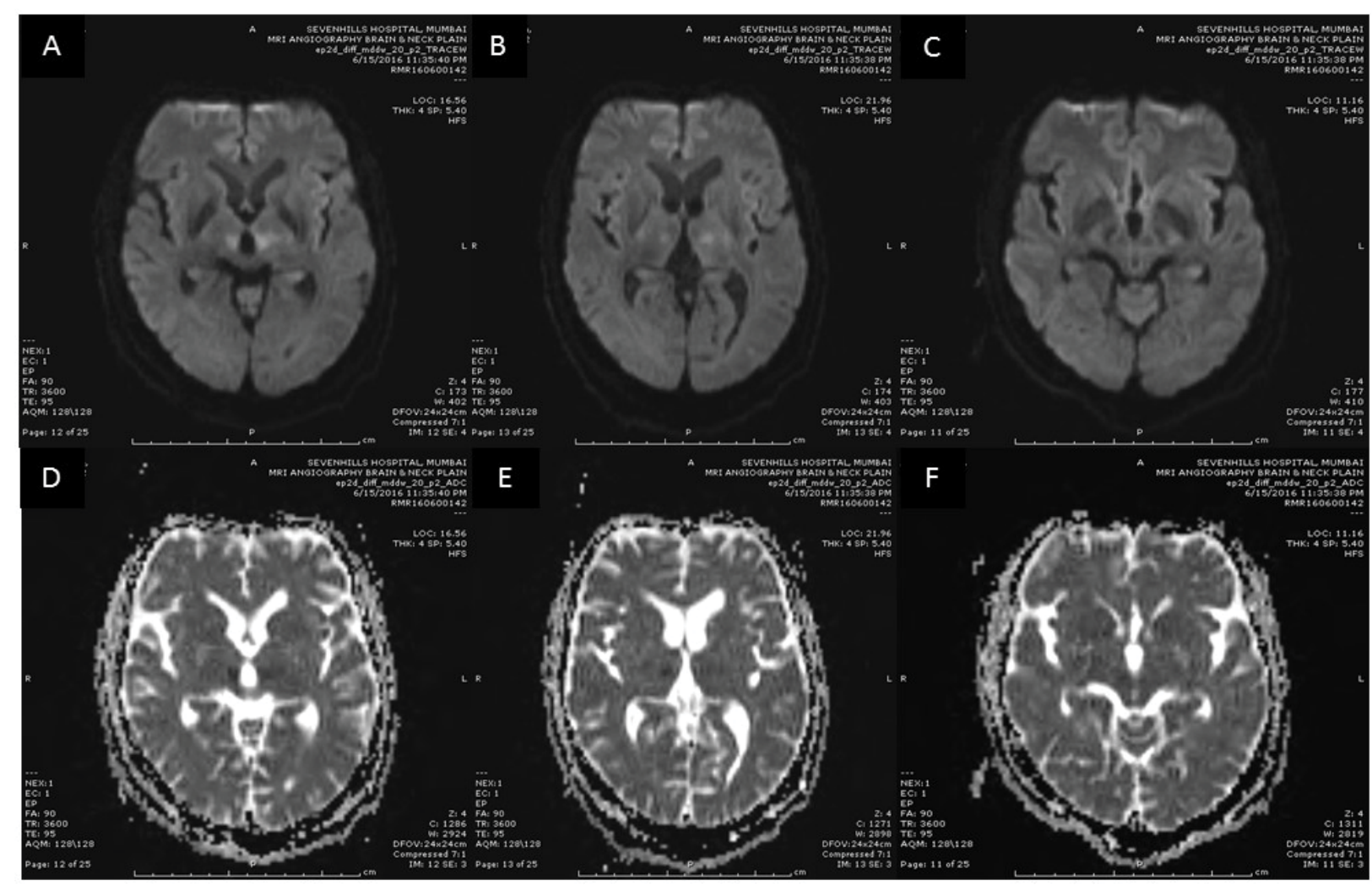

Figure 1 MRI brain DWI and ADC images on clinical presentation.

- A, B and C- Diffusion weighted images (DWI) showing hyper intensity in bilateral thalami with sparing of midbrain.

- $D, E$ and $F$ - apparent diffusion coefficient (ADC) showing hypo intensities (low $A D C$ ) in corresponding areas. 


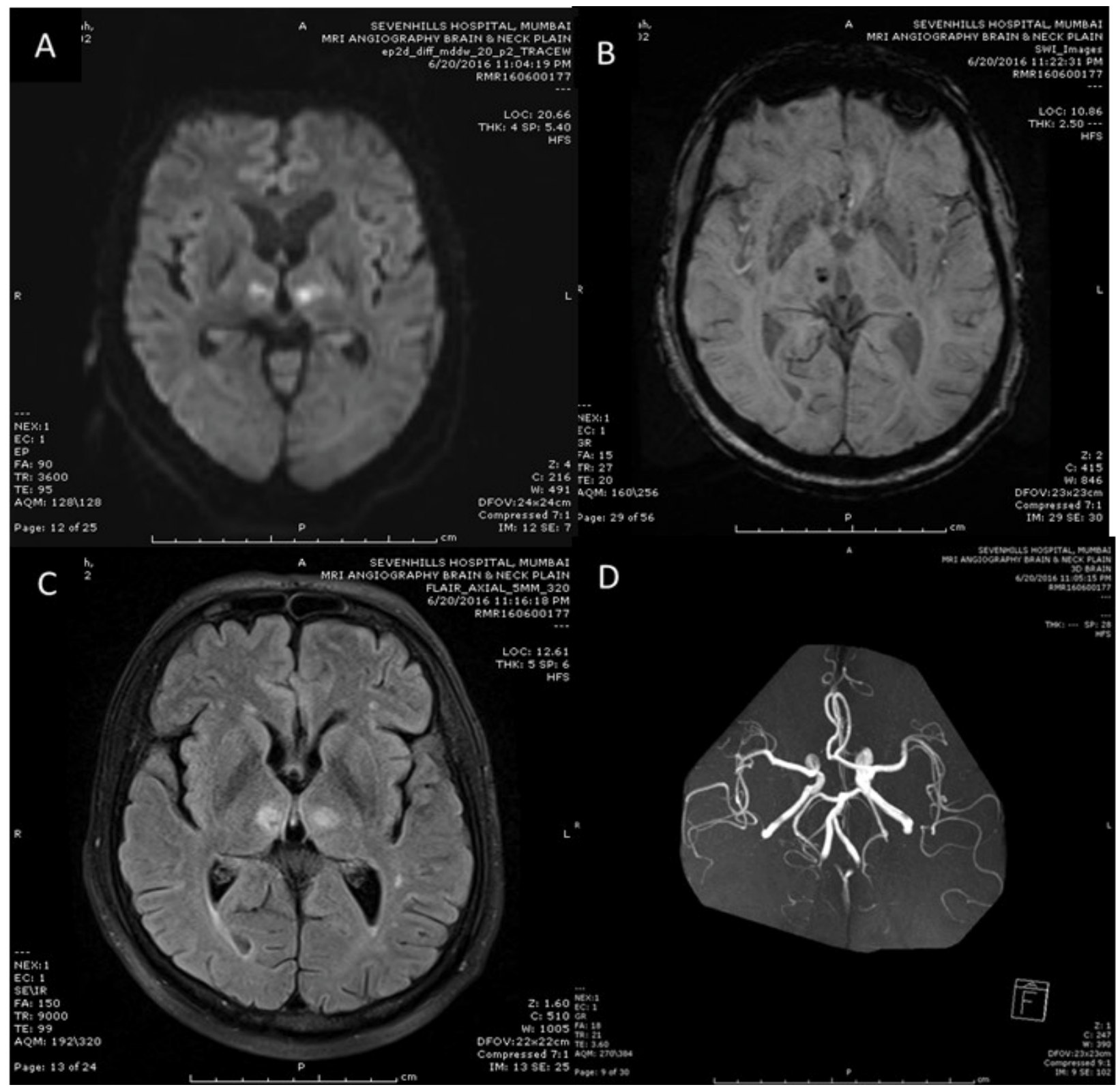

Figure 2 Follow up MRI on discharge.

- A-DWI images showing well-formed hyper intensities in bilateral thalami suggesting evolved infracts.

- B-Susceptibility weighted image (SWI) Showing subtle blooming in right thalamus suggesting hemorrhagic transformation.

- C-Fluid attenuated inversion recovery (FLAIR) sequence showing bilateral thalamic rounded hyper intensities.

- D-Intracranial time of flight TOF MRI angiogram showing normal flow related enhancement of vessels.

arising from the $\mathrm{P} 1$ segment of the PCA. Variant I is most common, in which each perforating artery arises from individual PCA. In variant II, bilateral perforating thalamic arteries arise from a single arterial trunk arising from P1 segment of PCA called the artery of Percheron. It supplies the Para median thalami and the rostral midbrain bilaterally. Variant III is an arcade variant, with several small perforating branches arising from a single arterial arc that bridges the P1 segments of both posterior cerebral arteries [5,6] (Figure 3).

The three cardinal clinical features include vertical gaze palsy (65\%), memory impairment (58\%) and coma (42\%) $[5,7]$.
Some cases patient may present with hemiplegia, ataxia and occulomotor disturbance suggesting bilateral thalamic infarction with rostral midbrain involvement (thalamopeduncular syndrome) [8]. A variety of clinical signs including dysarthria, impaired convergence, retraction of eyelids, amnesia, ataxia, involuntary movement of limbs have been described [9]. Our case initially presented with altered sensorium in the form of stupor and skew deviation. On improvement of sensorium, patient had amnesia for the event with occulomotor disturbances, vertical gaze palsy with some gait ataxia which improved with time. Altered consciousness can be explained by involvement of 

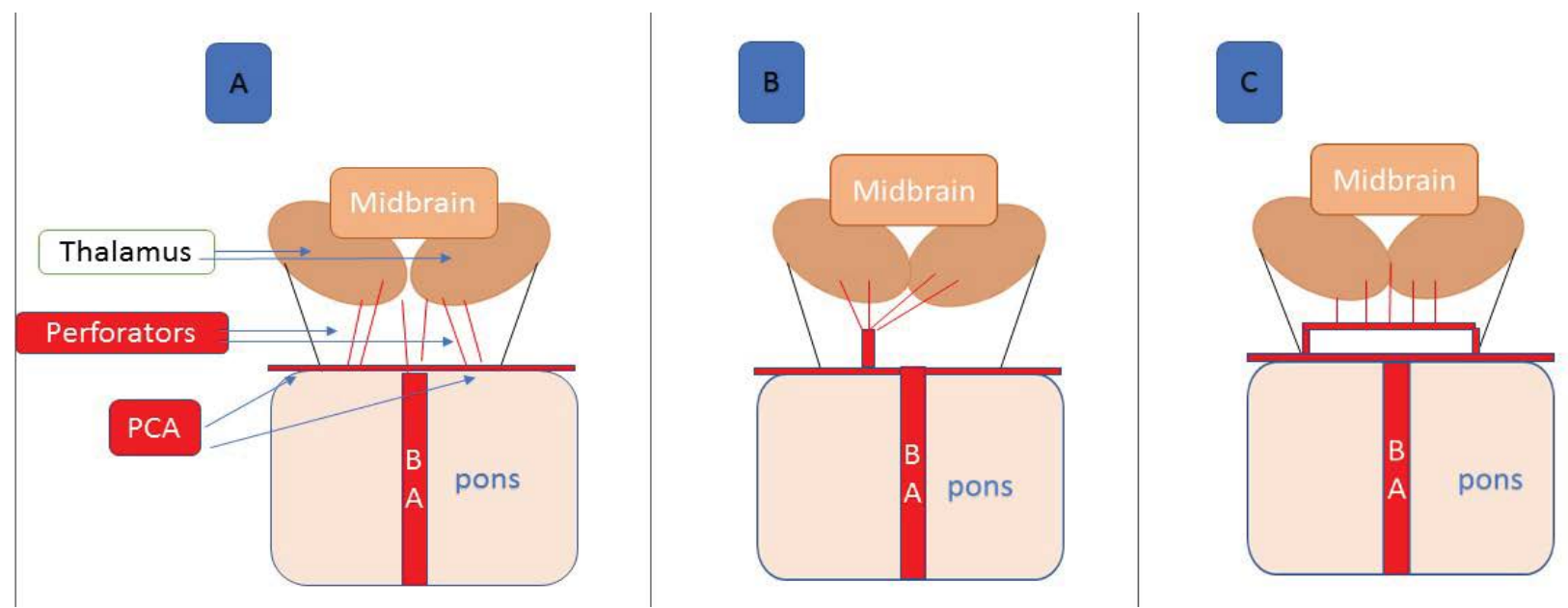

Figure 3 Anatomic variations in blood supply of thalamus and midbrain.

A) Multiple perforators arising from P1 segment of both posterior cerebral artery (PCA).

B) Perforators arising from single arterial trunk of P1 segment of one of the PCA (named as artery of Percheron AOP).

C) Multiple perforators arising from an artery bridging P1 segment of both PCA.

reticular activating system [10] and the disrupted connections between the thalamus and the anterior, orbitofrontal and medial prefrontal cortices. Vertical gaze palsy is due to disruption of the cortical input that traverses the thalamus to reach the rostral interstitial medial longitudinal fasciculus [11]. Mechanism for occlusion of artery of percheron includes atherothrombotic, cardioembolic, small vessel disease, vasculitis and rarely hypercoagulable state. Most common etiology for AOP infarction is cardio embolism followed by Cerebro-vascular small vessel disease [12]. In our case, we couldn't demonstrate any cardiac source of embolism and presumed etiology was cerebrovascular small vessel disease due to the vascular risk factors. MRI brain with diffusion-weighted imaging (DWI) is the ideal mode of imaging to diagnose infarction early [13] in most situations. However, many studies report normal imaging results in early CT and MR (DWI) brain scan in symptomatic and even comatose patients with AOP ischemic infarction [5]. Lazzaro et al. described 4 patterns of infarction: bilateral Para median thalamic with rostral midbrain (43\%); bilateral Para median thalamic without midbrain (38\%); bilateral Para median and anterior thalamic with midbrain (14\%); and, bilateral Para median and anterior thalamic without midbrain (5\%) infarctions. Our patient had bilateral Para median thalamic infarct without midbrain involvement. MR angiogram is essentially normal in AOP infarction. Even catheter

\section{References}

1 American College of Emergency Physicians (1999) Clinical policy for the initial approach to patients presenting with altered mental status. Ann Emerg Med 33: 251-328.

2 Percheron G (1973) The anatomy of the arterial supply of the human thalamus and its use for the interpretation of the thalamic vascular pathology. J Neurol 205: 1-13. angiography usually cannot demonstrate AOP [14]. Prognosis depends upon the baseline clinical status at presentation, extent of infarction and most importantly whether patient received timely thrombolytic treatment. Such patients are shown to show faster clinical recovery. $50 \%$ of patients have residual disability at the end of 6 months follow up [12]. Our case couldn't be subjected to thrombolytic therapy as he had active Gl bleed and presented outside the window period. Recovery was gradual with residual deficit which could have been prevented had the patient presented in window period without any contraindications for thrombolysis.

\section{Conclusion}

Acute onset altered mental status is a clinically challenging situation due to varied presentation with little signs to elicit and narrow therapeutic window. Also there are numerous causes to watch out for. The diagnosis of AOP infarction may be delayed due to atypical presentation and the therapeutic window for thrombolytic therapy can be missed which can result in significant neurological impairment. For patients presenting with sudden altered mental status, a differential diagnosis of AOP infarct must be considered and urgent MRI brain with DWI sequencing should be done for early diagnosis and treatment in the form of thrombolytic therapy which could result in good clinical recovery.

3 Kostanian V, Cramer SC (2007) Artery of Percheron Thrombolysis. AJNR Am J Neuroradiol 28: 870-871.

4 Xiao HY, Wang YX, Xu TD (2012) Evaluation and treatment of altered mental status patients in the emergency department: Life in the fast lane. World J Emerg Med 3:4.

5 Cassourret G, Prunet B, Sbardella F, Bordes J, Maurin O, et al. (2010) Ishemic stroke of the artery of Percheron with normal initial MRI: A case report. Case Rep Med 2: 425-734. 
6 Amin OSM, Shwani SS, Zangana HM, Hussein EMH, Ameen NA (2011) Bilateral infarction of paramedian thalami: a report of two cases of artery of Percheron occlusion and review of the literature. BMJ Case Rep 3.

7 Lazzaro NA, Wright B, Castillo M, Fischbein NJ, Glastonbury CM, et al. (2010) Artery of Percheron infarction: Imaging patterns and clinical spectrum. AJNR Am J Neuroradiol 31: 1283-1289.

8 Waterston JA, Stark RJ, Gilligan BS (1987) Paramedian thalamic and mid brain infarction: The mesencephalothalamic syndrome. Clin Exp Neurol 24: 45-53.

9 Reilly M, Connolly S, Stack J, Martin EA, Hutchinson M (1992) Bilateral paramedian thalamic infarction a distinct but poorly recognized syndrome. QJ Med 82: 63-70.

10 Krampla W, Schmidbauer B, Hruby W (2008) Ischaemic stroke of the artery of Percheron. Eur Radiol 18: 192-194.
11 López-Serna R, González-Carmona P (2009) Bilateral thalamic stroke due to occlusion of the artery of Percheronn in a patient with patent foramen ovale: A case report. J Med Case Reports 3: 7392.

12 Aaron S, Mani S, Prabhakar AT, Karthik K, Patil AB, et al. (2015) Stuck with a drowsy patient, evoke the Percheron. Neurol India 63: 542-547.

13 Raphaeli G, Liberman A, Gomori JM, Steiner I (2006) Acute bilateral paramedian thalamic infarcts after occlusion of the artery of Percheron. Neurology 66: E7.

14 Yarmohammadi H, Carasca A, Hsu DP (2011) Patent foramen ovale associated with the unusual presentation of unilateral paramedian thalamic perforating artery infarction after embolic occlusion of artery of Percheron: A case report and review of the literature. J Neurointerv Surg 3: 156-159. 